\title{
Aeromonas en la diarrea aguda de niños menores de 5 años
}

\author{
JOSÉ MARÍA GUEVARA D ${ }^{1}$, CARLOS HUAMANÍ2 ${ }^{2}$, RITO ZERPA ${ }^{1,3}$, ESTHER VALENCIA $^{1}$, \\ JOSÉ M-M GUEVARA G G,4, MARCIAL ANAYA ${ }^{5}$ y col. \\ ${ }^{1}$ Instituto de Medicina Tropical "Daniel A. Carrión". ${ }^{2}$ Hospital Docente Materno Infantil San Bartolomé. \\ ${ }^{3}$ Instituto de Salud del Niño. ${ }^{4}$ Hospital Nacional Daniel A. Carrión. ${ }^{5}$ Hospital Nacional Arzobispo Loayza.
}

\begin{abstract}
RESUMEN
OBJETIVO: Determinar la importancia de Aeromonas como agente diarreogénico en niños menores de 5 años, así como comparar su frecuencia con la de otros enteropatógenos. MATERIAL Y MÉTODOS: Entre 1998 y 1999, se tomó 285 muestras de heces de niños con diarrea aguda, en 5 Centros Hospitalarios, las que fueron enviadas al Instituto de Medicina Tropical "Daniel A. Carrión" en el medio de transporte Cary Blair, para su procesamiento e identificación de Aeromonas por el método Aerokey II. RESULTADOS: Aeromonas fue la bacteria más aislada entre los enteropatógenos, sobre todo en niños menores de 2 años y en verano, y la Aeromonas caviae fue la especie más frecuente. Aeromonas fue aislada en la mayoría de los casos del agar TCBS, por lo que empleamos discos de O/129 para diferenciarla de Vibrio cholerae. Todas las Aeromonas fueron sensibles in vitro a furazolidona y neomicina. CONCLUSIONES: Aeromonas fue el principal agente etiológico de la diarrea aguda acuosa, en niños menores de 5 años.
\end{abstract}

Palabras clave: Aeromonas; diarrea; enfermedades transmisibles; mortalidad infantil.

\section{Aeromonas IN CHILDREN LESS THAN 5 YEAR-OLD WITH ACUTE DIARRHEA SUMMARY}

OBJECTIVE: To determine Aeromonas importance as diarrhea agent in children less than 5 yearold and compare its frequency with other enteropathogens. MATERIAL AND METHODS: Between 1998 and 1999285 fecal samples were taken from children with acute diarrhea in 5 hospitals, which were then sent to "Daniel A. Carrión" Institute of Tropical Medicine in Cary Blair's transportation medium for processing by Aerokey II method. RESULTS: Aeromonas was the most frequent bacteria isolated among enteropathogens, mainly in children less than 2-year old and in summer, and Aeromonas caviae was the most frequent species. Aeromonas was mainly isolated in agar TCBS, employing O/129 disks to differentiate it from Vibrio cholerae. All Aeromonas were sensitive in vitro to furazolidone and neomycin. CONCLUSIONS: Aeromonas was the main etiological agent of aquous acute diarrhea in children less than 5 year-old.

Key words: Aeromonas; diarrhea; communicable diseases; infant mortality.

\section{INTRODUCCIÓN}

La diarrea aguda en niños menores de 5 años es una de las principales causas de morbimortalidad en nuestro medio. Las madres, de acuerdo

Correspondencia:

Dr. José María Guevara Duncan

Instituto de Medicina Tropical "Daniel A. Carrión"

José Santos Chocano 199.

Callao 2, Perú

E-mail: jomaguedu34@hotmail.com a la gravedad del caso, acuden a la farmacia, a la Posta Médica o al Hospital.

Los agentes etiológicos de la diarrea infecciosa pueden ser bacterias o virus. Las bacterias invasivas provocan una reacción inflamatoria en las heces, es decir, estimulan la presencia de leucocitos, a veces acompañados de hematíes. Los virus no producen reacción inflamatoria y su diagnóstico se realiza por microscopia electrónica y pruebas inmunológicas, que son costosas. 
Las Aeromonas son bacterias acuáticas, de agua dulce, que desde comienzos del siglo pasado fueron identificadas como causantes de procesos infecciosos en los animales acuáticos: anfibios, reptiles, peces, caracoles y otros $\left({ }^{1}\right)$.

Los microorganismos del género Aeromonas son patógenos oportunistas para el hombre. Han sido asociados con infecciones de heridas, particularmente después de la exposición del huésped al medio ambiente acuático; y, en los últimos años, algunas especies de este género están siendo reconocidas como potenciales agentes enteropatógenos, habiéndose demostrado producción de enterotoxinas y citotoxinas $\left({ }^{2-4}\right)$.

Las evidencias sobre el papel causal de estos microorganismos en la diarrea aguda han estado limitadas, pues son pocos los laboratorios que cumplen técnicas específicas y de fácil realización para la clasificación en especies de estos microorganismos. En 1991, Amy Carnahan diseña un nuevo método, llamado Aerokey II, en el cual, basado en sólo siete pruebas bioquímicas, fácilmente ejecutables en cualquier laboratorio de microbiología clínica, se logra la identificación de hasta 7 especies $\left({ }^{2,5}\right)$.

El objetivo de este estudio es esclarecer la importancia de Aeromonas como agente diarreogénico en niños menores de 5 años, así como comparar su frecuencia con la de otros enteropatógenos.

\section{MATERIAL Y MÉTODOS}

Durante los años 1998 y 1999 se tomó muestras de heces a niños que llegaron por diarrea, en los siguientes centros hospitalarios:

- 115 en el Hospital Docente Materno Infantil San Bartolomé.

- 58 en el Hospital Arzobispo Loayza.

- 54 en el Hospital Daniel A. Carrión.

- 48 en el Instituto de Salud del Niño.

- 10 en otros Centros.
En total, 285 muestras de igual número de pacientes.

Se llenó una ficha con cada caso, con datos previamente establecidos con los médicos pediatras.

Las heces, recientemente emitidas, fueron colocadas en un recipiente de plástico limpio, con la ayuda de una cucharita, y guardadas en refrigeración. Simultáneamente, se impregnó de heces un hisopo estéril y se los colocó en un tubo con medio de transporte Cary Blair, taponado herméticamente, el cual quedó a medio ambiente.

Periódicamente, las muestras eran recogidas y llevadas al Instituto de Medicina Tropical "Daniel A. Carrión", para ser procesadas de la siguiente manera: Con el hisopo contenido en el medio de transporte Cary Blair, se sembró en agar MacConkey, agar SS, agar TCBS, caldo selenito, agua peptonada alcalina y agar manitol salado, procediéndose a la incubación a $37^{\circ} \mathrm{C}$ por 24 horas, para luego hacer las resiembras correspondientes y el estudio bioquímico diferencial. Se hacía el estudio serológico cuando era requerido y, al identificarse una bacteria enteropatógena, se procedió al antibiograma con el método de Bauer y Kirby, con los siguientes antimicrobianos: ampicilina, acido nalidíxico, neomicina, cotrimoxazol y furazolidona.

El examen microscópico directo se realizó con la muestra obtenida en los recipientes de plástico.

En el caso específico de Aeromonas, para su identificación se empleó la prueba de oxidasa, discos vibriostáticos O/129 y el método llamado Aerokey II $\left(^{5}\right)$.

No se investigó Campylobacter ni virus.

\section{RESULTADOS}

De las 285 muestras de igual número de pacientes, con relación a la edad, 167 tenían de 0 
a 1 año, 83 de 1 a 2 años, 17 de 2 a 3 años, 5 de 3 a 4 años y 5 de 4 a 5 años. El 79\% vivía en casa que tenía agua potable y desagüe y el $21 \%$ no tenía.

Leucocitos en las heces tuvieron 68 pacientes $(24 \%)$ y no tuvieron leucocitos 217 pacientes $(76 \%)$. Cuarenta muestras tuvieron de 1 a 2 cruces y 28 de 3 a 4 cruces.

De las 40 muestras que tuvieron 1 a 2 cruces, a $27(67,5 \%)$ no se le aisló alguna bacteria diarreogénica y en $12(30 \%)$, se aisló las siguientes bacterias: 4 Shigellas, 3 Aeromonas, 4 Staphylococcus aureus y 1 Salmonella.

De las 28 muestras que tuvieron 3 cruces, a $16(57 \%)$ no se le aisló alguna bacteria diarreogénica y en 12 (43\%) se aisló las siguientes bacterias: 6 Shigellas, 3 Staphylocccus aureus, 2 Aeromonas y 1 Escherichia coli enteropatógeno.

De las 217 muestras que no tuvieron leucocitos, en 177 (82\%) no se aisló bacterias diarreogénicas y en las $40(18 \%)$ restantes, se aisló: 17 Aeromonas, 8 Staphylococcus aureus, 7 Salmonellas, 5 Shigellas, 2 Escherichia coli capsulada (observación personal) y 1 Escherichia coli enteropatógeno.

De las 285 muestras, 89 (31\%) demostraron alteración en su flora normal, lo cual podría indicar que esos pacientes estaban recibiendo algún tratamiento antimicrobiano.

En la Tabla 1, se observa en orden decreciente las bacterias diarreogénicas aisladas de todas las muestras.

Con relación a Aeromonas, se aisló 22 cepas de 20 pacientes, porque en dos casos se identificó dos diferentes especies de Aeromonas.

La edad de los niños estuvo entre los 2 meses a 2 años. Catorce fueron de sexo masculino y 6 del femenino.

Tres aislamientos de Aeromonas se produjo en niños que tuvieron una y dos cruces de
Tabla 1.- Bacterias diarreogénicas aisladas de todas las muestras.

\begin{tabular}{ll}
\hline Aeromonas & 22 \\
Shigella & 15 \\
Staphylococcus aureus & 15 \\
Salmonellas & 08 \\
E. coli enteropatógeno & 02 \\
E. coli capsulado (observación personal) & 02 \\
\hline
\end{tabular}

leucocitos en heces; 2 aislamientos fueron de heces con tres cruces de leucocitos y 15 aislamientos de heces que no tuvieron leucocitos.

Tenían agua y desagüe en su casa 15 niños, mientras que 5 no tenían.

El tiempo de enfermedad al ser atendidos osciló entre 12 horas y 8 días. El número de evacuaciones en 24 horas fue de 4 a 10 veces.

Naúsea tuvieron 10 niños; fiebre 10 niños; dolor abdominal 8 niños. Deshidratación leve tuvieron 16 de los casos, mientras que 4 tuvieron deshidratación moderada.

La mayoría de los aislamientos se produjo en verano, con 12 casos, en primavera 4 , en otoño 3 y en invierno uno.

Quince de los aislamientos de las 22 cepas de Aeromonas se realizó en el agar TCBS, 5 después del enriquecimiento en agua peptonada alcalina (APW) y resiembra en agar MacConkey y 2 después del enriquecimiento en APW y resiembra en agar TCBS.

En la Tabla 2, se muestra las especies que se identificó, de los cuales:

En dos casos, Aeromonas estuvo acompañada con Staphylococcus aureus y, en los demás, estuvo Aeromonas como única bacteria diarreogénica.

Todas las Aeromonas fueron sensibles a furazolidona y neomicina, $18(81 \%)$ a ácido nalidíxico y cotrimoxazol y 15 (68\%) a 
Tabla 2.- Especies de Aeromonas identificadas.

$\begin{array}{ll}\text { A. caviae } & 16 \\ \text { A. veronii biovar sobria } & 03 \\ \text { A. hydrophila } & 02 \\ \text { A. schubertii } & 01\end{array}$

ampicilina .

\section{DISCUSIÓN}

Aeromonas es un reconocido microorganismo causante de diarrea aguda, sobre todo en niños menores de 2 años $\left({ }^{6}\right)$, tal como lo hemos confirmado.

Los estudios epidemiológicos sobre la función etiológica de Aeromonas en la diarrea está limitada, pues son pocos los laboratorios de microbiología que emplean técnicas y métodos específicos para su aislamiento e identificación $\left({ }^{7}\right)$.

Las especies del género Aeromonas son consideradas habitantes autóctonos de ambientes acuáticos no salinizados $\left({ }^{8}\right)$, por lo que son importantes en el control de calidad del agua de beber $\left({ }^{9}\right)$. Tiene un ciclo estacional, con baja densidad en los meses fríos y con incremento en el riesgo de infección en los meses de verano $\left({ }^{10,11}\right)$.

En nuestro estudio hemos encontrado a Aeromonas como la más frecuente entre otros enteropatógenos, en niños menores de 2 años, sobre todo en los meses de verano -como lo reporta también M. Gracey y col- $\left({ }^{12}\right)$, independientemente del sexo del niño y tener o no agua y desagüe en su casa, siendo el cuadro clínico inespecífico.

Las infecciones son frecuentemente adquiridas como resultado de una exposición directa al agua que alberga estos microorganismos $\left({ }^{13}\right)$; la diarrea que causa produce una deshidratación leve, conforme a nuestras observaciones.
Los leucocitos fecales son de utilidad muy limitada en la práctica diaria. Se correlaciona con la presencia de una bacteria invasora sólo cuando están presentes en alto número $(+++)$. Pero, en esta situación ya es posible observar pus, e incluso sangre, a simple vista. Si los leucocitos fecales son $(+)$ a $(++)$, el examen pierde inmediatamente especificidad. Se puede apreciar presencia de moderada cantidad de leucocitos fecales en diarrea por agentes no invasores, incluyendo el rotavirus, probablemente como manifestación microscópica de una inflamación química del área rectal o perianal $\left({ }^{14}\right)$. Se encuentra ocasionalmente Aeromonas como invasora; dos de nuestros pacientes tuvieron $(+++)$ de leucocitos, mientras la mayoría de los casos no tuvo leucocitos, como es lo frecuente.

Existe un medio de cultivo con ampicilina para el aislamiento, sobre todo, de Aeromonas hydrophila $\left({ }^{15}\right)$. En nuestro estudio aislamos dos Aeromonas hydrophila: una sensible y la otra resistente a la ampicilina. Además, las Aeromonas en total fueron menos sensibles, en el antibiograma, a la ampicilina, por lo que no sería recomendable emplear el medio de cultivo con ampicilina.

El agar TCBS es un medio selectivo para aislar Vibrio, pero Aeromonas logra desarrollar frecuentemente en él, como en nuestro caso: de 22 cepas, 17 fueron aisladas en el agar TCBS, dos de ellas después de enriquecimiento en APW $\left({ }^{15-17}\right)$.

En el agar TCBS, las colonias de Aeromonas se las aprecia de color amarillo, igual que Vibrio cholerae, porque ambas metabolizan la sacarosa que contiene el medio. Bioquímicamente, se parecen mucho Aeromonas y $V$. cholerae, por lo que es indispensable el empleo de la sustancia vibriostática en discos conocida como $\mathrm{O} / 129$, a la cual es sensible $V$. cholerae y resistente Aeromonas.

La especie que más aislamos (16 cepas) fue 
Aeromonas caviae, lo cual es también reportado por T. Teka y col $\left({ }^{18}\right)$.

Todas las Aeromonas fueron susceptibles a furazolidona y neomicina, por lo que podrían ser empleados en el tratamiento de la diarrea aguda en niños menores de 5 años, causada por esta bacteria.

\section{AGRADECIMIENTOS}

El trabajo se hizo con el financiamiento de la Universidad Nacional Mayor de San Marcos, en un programa de ayuda a la investigación.

A la Sra Victoria Molina, Jefa de Enfermeras del Servicio de Emergencia en Hidratación Oral del Hospital Docente Materno-Infantil San Bartolomé, quien nos ayudó mucho en la obtención de las muestras.

A la Srta. Nelly Llerena, Técnica del Hospital Guillermo Almenara, quien nos ayudó a realizar los cuadros finales.

Al NMRCD (Srta. Rina Meza) que nos proporcionó los discos de $\mathrm{O} / 129$.

\section{BIBLIOGRAFÍA}

1. Hazen TC, Fliermans CB, Hirsch RP, Esch GW. Prevalence and distribution of Aeromonas hydrophila in the United States. Aplied and Enviromental Microbiology 1978; 36(5): 731-8.

2. Bravo L, Monté RJ, Cabrera AV, Gómez N, Hernández M, García B. Nuevas especies de Aeromonas aisladas en Cuba. Rev Cubana Med Trop 1995; 47(3): 215-6.

3. Trower CJ,Abo S, Majeed KN, von Itzstein M. Production of an enterotoxin by a gastro-enteritis-associated Aeromonas strain. J Med Microbiol 2000; 49(2): 121-6.

4. Brandi G, Sisti M, Giardini F, Shiavano GF, Albano A. Survival ability of cytotoxic strains of motile Aeromonas spp. in different types of water. Letters Applied Microbiol 1999; 29(4): 211-5.

5. Carnahan AM, Behram S, Joseph SW. Aerokey II: a flexible key for identifying clinical Aeromonas especies. J Clin Microbiol 1991; 29: 2843-9.

6. Albert M J, Faruque AS, Faruque SM, Sack RB, Mahalanabis D. Case-control study of enteropathogens associated with childhood diarrhea in Dhaka, Bangladesh. J Clin Microbiol 1999; 37(11): 3458-64.

7. Bravo L, Monté RJ, Abad R, García B. Determinación de especies del género Aeromonas procedentes de alimentos y aguas residuales. Rev Cubana Aliment Nutr 1992; 6(2): 108-11.

8. Kaper JB, Lockman H, Colwell RR. Aeromonas hydrophila: ecology and toxigenicity of isolates from an estuary. J Appl Bacteriol 1981; 50: 359-77.

9. Sixl W, Sixl K, Sixl-Voigt B. Quality of water-quality of life. Central Eur J Public Health 1999; 7(4): 216-20.

10. Hitorami H, Sese C, Kagawa H. Correlations of Aeromonas hydrophila with indicator bacteria of water quality and enviromental factors in a mountain stream. Water Environment Research Alexandria 1999; 71(2): 132.

11. Janda JM, Bottone E J, Skinner CV, Calcaterra D. Phenotypic markers associated with gastrointestinal Aeromonas hydrophila isolates from symptomatic children. J Clin Micobiol 1983; 17(4): 588-91.

12. Gracey M, Burke V, Rockhill RC, Sunoto S. Aeromonas species as enteric pathogens. Lancet 1982; 23: 223-4.

13. Daily OP, Joseph SW, Coolbaugh JC, Walker RI, Merrel BR, et al. Association of Aeromonas sobria with human infection. J Clin Microbiol 1981; 13(4): 769-77.

14. Guiraldes E, Larraín, Harris P. Diarrea aguda en la infancia. http://escuela.med.puc.cl/Departamentos/Pediatria/ Pediat.2030html.

15. Moulsdale MT. Isolation of Aeromonas from faeces. Lancet 1983; 12: 351.

16. Shread P, Donovan TJ, Lee JV. A survey of the incidence of Aeromonas in human faeces. Soc Gen Microbiol Quart 1981; 8: 184.

17. Von Graevenitz A, Bucher C. Evaluation of differential and selective media for isolation of Aeromonas and Plesiomonas spp. from human feces. J Clin Microbiol 1983; 17(1): 16-21.

18. Teka T, Faruque AS, Hossain MI, Fuchs GJ. Aeromonasassociated diarrhoea in Bangladeshi children: clinical and epidemiological characteristics. Ann Trop Paediatrics 1999; 19(1): 15-20. 